Mass

Spectrometry 


\section{CLINICAL BIOCHEMISTRY}

\section{PRINCIPLES - METHODS}

APPLICATIONS

1

Series Editors

H.Ch.Curtius

M.Roth

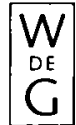

Walter deGruyter

Berlin · NewYork

1989 


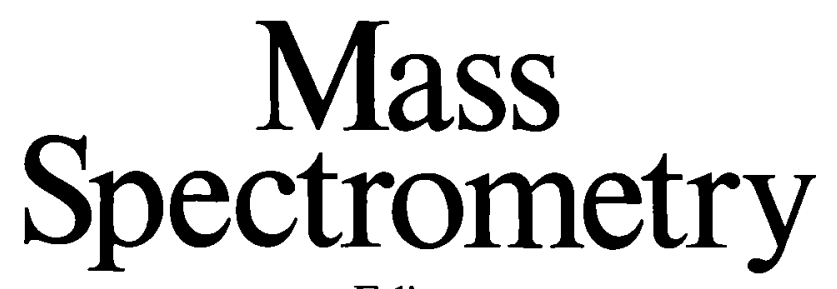

Editor

A.M.Lawson

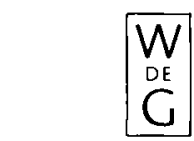

Walter deGruyter

Berlin - NewYork

1989 
Editor

Dr. Alexander M. Lawson

Head, Section of Clinical Mass Spectrometry

Clinical Research Centre

Watford Road

Harrow

GB-Middlesex HA1 3UJ

Great Britain

Series Editors

Dr. Hans-Christoph Curtius

Head, Division of Clinical Chemsitry

Department of Pediatrics

University of Zurich

Steinwiesstrasse 75

Dr. Marc Roth

Director, Central Laboratory of

Clinical Chemistry

CH-8032 Zurich

Cantonal Hospital

Switzerland

University of Geneva

CH-1211 Geneva 4

Switzerland

Publishers

Walter de Gruyter \& Co.

Walter de Gruyter, Inc.

Genthiner Straße 13

200 Saw Mill River Road

D-1000 Berlin 30

Fed. Republic of Germany

Hawthorne, N.Y. 10532

Telephone (030) 26005-0

U.S.A.

Telefax (030) 260 05-251 · Telex 184027

Telephone (914) 747-0110

Telefax (914) 747-1326 · Telex 646677

\section{Library of Congress Cataloging in Publication Data}

\section{Mass spectrometry.}

(Clinical biochemistry ; 1)

Includes bibliographies and index.

1. Mass spectrometry. 2. Clinical biochemistry-Methodology. I.

Lawson, A. M. II. Series: Clinical biochemistry (Berlin, Germany) ;

I. [DNLM: 1. Spectrum Analysis, Mass. QC 454.M3 M4141]

QP519.9.M3M35 $1989 \quad 543^{\prime} .0873 \quad 88-33414$

ISBN 0-89925-488-8 (U.S.)

Deutsche Bibliothek Cataloging in Publication Data

Mass spectrometry / ed. A. M. Lawson. - Berlin ; New York :

de Gruyter, 1989

(Clinical biochemistry ; 1)

Mit 186 ill. u. 55 tab.

ISBN 3-11-007751-5

NE: Lawson, Alexander M. [Hrsg.]; GT

Copyright (C) 1988 by Walter de Gruyter \& Co., Berlin 30.

All rights reserved, including those of translation into foreign languages. No part of this book may be reproduced in any form - by photoprint, microfilm or any other means nor transmitted nor translated into a machine language without written permission from the publisher.

Typesetting and printing: Arthur Collignon GmbH, Berlin. Binding: Dieter Mikolai, Berlin.

Cover design: Hansbernd Lindemann, Berlin. - Printed in Germany. 